\section{Response frequency equalization: A bias model for psychophysics}

\author{
ALBERT ERLEBACHER and ROBERT SEKULER \\ Northwestern University, Evanston, Illinois 60201
}

A model for response bias in psychophysics is derived. Of the model's two aspects, one is concerned with the generation of sensory states and the other with rules for transforming sensory states into responses. The model incorporates the bias rule that Ss tend to use available responses with equal frequency. Applications to experiments which use the method of constant stimuli are discussed. Despite the contrary claims of Restle and Levison (1971), the model not only predicts their data quite well but also, under certain conditions, does so better than the theory of adaptation level used by Restle and Levison themselves.

The main concerns of psychophysics are "the laws that relate the responses of men and animals to the energetic configurations of the environment... [Stevens, 1958]." Unfortunate though it may be, factors quite unrelated to energetic configurations intervene in and shape the outcomes of psychophysical experiments. Among such factors are the expectations and response preferences which Ss bring to the laboratory. The possible intervention of such factors makes their identification and measurement a virtual prerequisite to the fulfillment of the main concern of psychophysics.

Contributing to this prerequisite, we have argued that the method of constant stimuli is susceptible to distortion by the response preferences of $\mathrm{S}$ (Sekuler \& Erlebacher, 1971). We have reinterpreted the results of Levison and Restle (1968) and those of Bell and Bevan (1968), proposing that the effects which both sets of investigators attributed to adaptation level (Helson, 1964) were more appropriately assigned to the tendency of $\mathrm{Ss}$ to use available responses with approximately equal frequency.

In their reply, Restle and Levison (1971) have presented models for both our position and theirs in the form of flow charts. We agree that their conceptualization of our model is essentially correct, and we assume that the model representing their position is also Although we remain unconvinced of the soundness of their position, we wish to set aside discussion of the logical-philosophical differences in our approaches.

The purpose of this paper is to present in a formal fashion the response frequency equalization (RFE) model which we proposed earlier. The purpose of doing so at this time largely is to correct some crrors in Restle and Levison's reply to our original cautionary note. Restle and Levison report an experiment which, they claim, would discriminate between their
Like many other contemporary models in psychophysics, the RFE model has two conceptually separable portions, one concerned with sensory processes and the other devoted to decision making. Although RFE theory may have strong implications for virtually all the psychophysical methods, we discuss it here in the restricted context of the method of constant stimuli.

\section{Sensory Process Portion of the Model}

On each of a number of trials, two stimuli are presented to $S$. The value of one, the standard stimulus (Std), is invariant from trial to trial, while the value of the second, comparison stimulus (Co), can take on any one of several values. The presentation of $\mathrm{Co}$ generates, in a probabilistic fashion, one of three possible sensory conditions. Beginning with Cos less than Std and going up to those greater than Std, we assume that the probability of the sensory state lesser decreases in a manner approximated by the cumulative normal distribution. Similarly, beginning with $\operatorname{Cos}$ of low magnitude and again working up, we assume that the probability of a sensory state greater increases in a manner approximated by the cumulative normal distribution. Figure 1 shows these relationships. In Fig. 1 we show the complement of $\operatorname{Pr}$ (lesser) rather than the probability itself so that both cumulative normals shown will be increasing functions of $\mathrm{Co}$ magnitude. For the sake of computational simplicity we assume that the standard deviations of these two cumulative normals are equal. The vertical distance between the two curves defines the probability that a given $\mathrm{Co}$ will produce yet a third sensory state, uncertainty.

In Fig. 1, $\operatorname{Pr}$ (greater), $\operatorname{Pr}($ lesser $)$, and Pr(uncertain) are denoted by $G, L$, and $U$, respectively. Also shown are several other features of the model which facilitate comparisons with traditional treatments of data from the method of constant stimuli. The abscissa values for which $\operatorname{Pr}$ (lesser) and $\operatorname{Pr}$ (greater) are equal to 0.5 are the lower $\left(T_{L}\right)$ and upper $\left(T_{U}\right)$ thresholds of change, respectively - the endpoints of the "interval of uncertainty." Half of the difference between these two is the difference

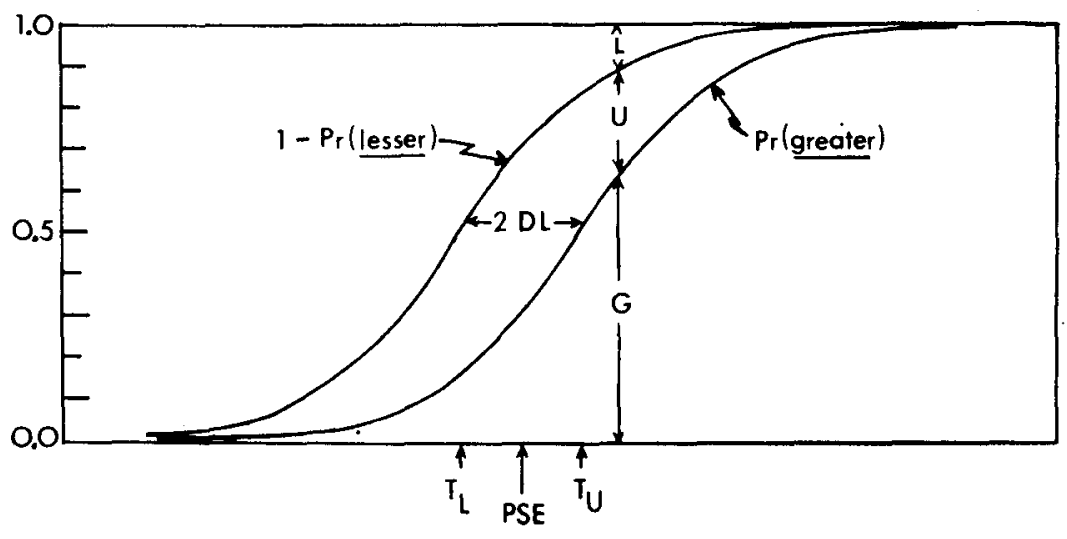

Fig. 1. Underlying psychometric functions for the RFE model. 

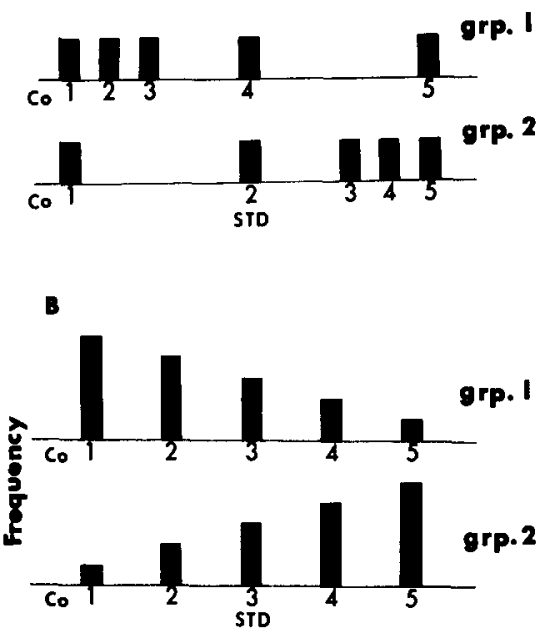

Fig. 2. Examples of variation in stimulus ensemble: (A) variation in stimulus distribution; (B) variation in stimulus frequency.

threshold (DL). The midpoint of the interval is the point of subjective equality (PSE). When the PSE is not equal to Std, the difference is the constant error.

\section{Response Bias Portion of the Model ${ }^{1}$}

We assume that $\mathrm{S}$ follows three simple rules for converting sensory states into overt responses: if the trial produces the state lesser, respond "Lesser"; if the trial produces the state greater, respond "Greater"; if the trial produces a state uncertain, make that response which will tend to equalize the frequency with which the two responses, "Lesser" and "Greater," will have been made. In the model, the probability that the state uncertain will occur depends upon the separation between the two cumulative normals. The separation depends on the standard deviations (or slopes) of the two functions and on their means, $T_{L}$ and $T_{U}$. These, then, are the only parameters of the RFE model.

\section{BASIC EXAMPLES}

Before applying the model in its complete detail, we wish to consider, for purposes of illustration, some intuitively obvious (or nearly so) derivations from RFE. The aspects of the model which are concerned with Pr(uncertain) are largely finessed and will be introduced in the following section.

\section{Variation in the Distribution of Cos}

- Consider a hypothetical experiment using two groups of Ss and the method of constant stimuli. Each group is given a different $\mathrm{Co}$ stimulus distribution. The distributions of $\operatorname{Cos}$ along the stimulus dimension are asymmetrical with respect to the Std and are mirror images of one another. Figure 2a shows these relationships in abbreviated form. For the sake of argument, assume that for Group 1, $\operatorname{Cos} 1,2$, and 3 are so placed with respect to Std that they virtually always lead to the state lesser and, hence, "Lesser" responses. Stimulus 5 is so placed as to yield nearly $100 \%$ greater states and "Greater" responses. Stimulus 4, at the value of Std, will generate an appreciable number of uncertain states which, because of the predominance of "Lesser" judgments, will be converted by response equalization into "Greater" judgments. This will inflate the proportion of "Greater" judgments, pulling the PSE to a stimulus value lower on the stimulus dimension than would have been found in the absence of response frequency equalization. This produces a negative constant error. The opposite occurs for Group 2 in our hypothetical experiment. Imagine that Co 1 for that group is so placed as to yield lesser states and "Lesser" responses virtually all the time. Comparison stimuli, 3, 4, and 5, are placed to yield greater states and "Greater" responses with near certainty. The remaining $\mathrm{Co}$, Number 2, will produce many uncertain states which will be converted into responses of "Lesser" in order to equalize the frequency with which the two responses are offered. This will artificially raise the PSE, producing a positive constant error. This example shows how RFE could produce opposite shifts in the PSEs of the two groups, much as $\mathrm{CAL}$ theory would predict. In this example, however, the shifts in PSE are produced by the operation of response bias rather than by any factor of adaptation level.

Differences in the Frequency with which Cos are Presented

Imagine another hypothetical method of constant stimuli experiment. The two groups are each presented with the same Cos, but the Cos are presented with unequal frequencies. The particular pattern of presentation frequencies is shown for the two groups in Fig. 2b. The frequency distributions are mirror images of one another. To simplify the interpretation, assume that all the stimuli except the middle Co are far enough away from Std to generate essentially no uncertain states for the response bias part of the model to operate upon. Because of the asymmetrical frequency distributions, Group 1 will have a predominance of "Lesser" responses, while Group 2 will have a predominance of "Greater" responses. According to the RFE model, Group 1 would tend to convert its uncertain states into "Greater" responses while Group 2 would convert its uncertain states into "Lesser" responses. These tendencies would shift the PSEs for the two groups in opposite directions, lowering that for Group 1 and raising that for Group 2. These effects, also, resemble those predicted from adaptation level theory. These examples show how each of two factors, Co distribution and unequal frequency of presentation, will interact with the RFE principle to alter the PSE.

\section{APPLICATION OF THE RFE MODEL 2}

Restle and Levison (1971) used the same set of asymmetrically distributed $\operatorname{Cos}$ for both their groups, varying the frequency of presentation of each stimulus so that the frequency distributions of the two groups were "mirror images." The stimuli used and their presentation frequencies are given in Table 1 . The major finding of the Restle and Levison experiment is that the PSE for Group 2 is slightly higher than that for Group 1. This relationship, they claim, is not in keeping with the RFE model which they term "logically possible" but "empirically untrue." If Restle and Levison were correct, our model would surely merit the distinction of being the most ephemeral model in psychophysics for the year 1971. Being born in one paper and being "laid to rest" just a few pages later

Table 1

Distribution of Stimulus Frequencies and Empirical Psychometric Functions for the Restle and Levison Experiment

\begin{tabular}{|c|c|c|c|c|c|}
\hline \multirow[b]{2}{*}{ Stimulus } & \multicolumn{2}{|c|}{ Presentation Frequency } & \multicolumn{3}{|c|}{ Proportion of "Greater" Responses } \\
\hline & Group 1 & Group 2 & Group 1 & Group 2 & Average \\
\hline 10.16 & 6 & 1 & .0145 & .0305 & .0225 \\
\hline 12.70 & 1 & 0 & .0186 & $\longrightarrow$ & .0186 \\
\hline 14.77 & 1 & 1 & .0435 & .0231 & .0333 \\
\hline 16.83 & 1 & 3 & .1375 & .0593 & .0984 \\
\hline 17.62 & 1 & 15 & .2795 & .1532 & .2164 \\
\hline 18.73 & 10 & 10 & .7074 & .5092 & .6083 \\
\hline 19.37 & 15 & 1 & .7928 & .7023 & .7475 \\
\hline 21.59 & 3 & 1 & .9875 & .9470 & .9673 \\
\hline 22.54 & 1 & 1 & .9812 & 1.0000 & .9906 \\
\hline 24.77 & 0 & 1 & $\longrightarrow$ & .9924 & .9924 \\
\hline 27.15 & 1 & 6 & .9870 & .9936 & .9903 \\
\hline
\end{tabular}


Table 2

Calculations for the Application of the RFE Model to the Restle and Levison Data

\begin{tabular}{|c|c|c|c|c|c|c|c|c|c|c|}
\hline $\begin{array}{l}\text { Stim- } \\
\text { ulus }\end{array}$ & $\begin{array}{c}\mathrm{Pr} \\
\text { (greater) }\end{array}$ & $\begin{array}{c}\mathrm{Pr} \\
\text { (lesserj }\end{array}$ & $\begin{array}{c}P_{r} \\
\text { (uncerrain) }\end{array}$ & $\mathbf{N}$ & $\stackrel{\mathrm{N}}{\text { (greater) }}$ & $\begin{array}{c}\mathrm{N} \\
\text { (lesser) }\end{array}$ & $\begin{array}{c}\mathrm{N} \\
\text { (uncertain) }\end{array}$ & $\begin{array}{c}\mathrm{N} \\
\text { (uncertain to } \\
\text { "Gredter") }\end{array}$ & $\begin{array}{c}\text { Adjusted } \\
\mathrm{N} \\
\text { (“Greater") }\end{array}$ & $\begin{array}{l}\text { Adjusted } \\
\text { Pr } \\
\text { ("Greater") }\end{array}$ \\
\hline \multicolumn{11}{|c|}{ Group 1} \\
\hline 10.16 & .0000 & .9971 & .0029 & 6 & .0000 & 5.9826 & .0174 & .0081 & .0081 & .0014 \\
\hline 12.70 & .0003 & .9469 & .0528 & 1 & .0003 & .9469 & .0528 & .0247 & .0250 & .0250 \\
\hline 14.77 & .0069 & .7513 & .2417 & 1 & .0069 & .7513 & .2417 & .1129 & .1198 & 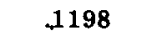 \\
\hline 16.83 & .0631 & .4000 & .5369 & 1 & .2631 & .4000 & .5369 & .2507 & .3138 & .3138 \\
\hline 17.62 & .1206 & .2707 & .6087 & 1 & .1206 & .2707 & .6087 & .2843 & .4049 & .4048 \\
\hline 18.73 & .2515 & .1328 & .6156 & 10 & 2.5150 & 1.3280 & 6.1560 & 2.8749 & 5.3899 & .5390 \\
\hline 19.37 & .3519 & .0803 & .5677 & 15 & 5.2785 & 1.2045 & 8.5155 & 3.9767 & 9.2552 & .6170 \\
\hline 21.59 & .7338 & .0080 & .2581 & 3 & 2.2014 & .0240 & .7743 & .3616 & 2.5630 & .8543 \\
\hline 22.54 & .8541 & .0023 & .1436 & 1 & .8541 & .0023 & .1436 & .0671 & .9212 & .9212 \\
\hline 24.77 & - & - & - & 0 & .0000 & .0000 & .0000 & .0000 & .0000 & $\square$ \\
\hline 27.15 & .9992 & .0000 & .0008 & 1 & .9992 & .0000 & .0008 & .0004 & .9996 & .9996 \\
\hline Total & & & & 40 & 12.0391 & 10.9103 & 17.0477 & 7.9614 & 20.0005 & \\
\hline \multicolumn{11}{|c|}{ Group 2} \\
\hline 10.16 & .0000 & .9971 & .0029 & 1 & .0000 & .9971 & .0029 & .0010 & .0010 & .0010 \\
\hline 12.70 & - & - & - & 0 & .0000 & .0000 & .0000 & .0000 & .0000 & $\square$ \\
\hline 14.77 & .0069 & .7513 & .2417 & 1 & .0069 & .7513 & .2417 & .0875 & .0944 & .0944 \\
\hline 16.83 & .0631 & .4000 & .5369 & 3 & .1893 & 1.2000 & 1.6107 & .5831 & .7724 & .2575 \\
\hline 17.62 & .1206 & .2707 & .6087 & 15 & 1.8090 & 4.0605 & 9.1305 & 3.3052 & 5.1142 & .3409 \\
\hline 18.73 & .2515 & .1328 & .6156 & 10 & 2.5150 & 1.3280 & 6.1560 & 2.2285 & 4.7435 & .4744 \\
\hline 19.37 & .3519 & .0803 & .5677 & 1 & .3519 & .0803 & .5677 & .2055 & .5574 & .5574 \\
\hline 21.59 & .7338 & .0080 & .2581 & 1 & .7338 & .0080 & .2581 & .0934 & .8272 & .8272 \\
\hline 22.54 & .8541 & .0023 & .1436 & 1 & .8541 & .0023 & .1436 & .0520 & .9061 & .9061 \\
\hline 24.77 & .9805 & .0001 & .0195 & 1 & .9805 & .0001 & .0195 & .0071 & .9876 & .9876 \\
\hline 27.15 & .9992 & .0000 & .0008 & 6 & 5.9952 & .0000 & .0048 & .0017 & 5.9969 & .9995 \\
\hline Total & & & & 40 & 13.4357 & 8.4276 & 18.1355 & 6.5650 & 20.0007 & \\
\hline
\end{tabular}

hardly constitutes longevity. However, we have found that the observations of Restle and Levison on the empirical validity of our model are themselves invalid. But, rather than speculate on the various sources of confusion among the four of us, we will present our attempts to run the Restle and Levison experiment through our model. To anticipate the result somewhat, our model, over a wide range of parameters, provides an excellent approximation to the actual outcome reported by Restle and Levison. This demonstrates that our model is not only "logically possible," as Restle and Levison acknowledged, but also "empirically true."

The steps involved in the application of RFE are as follows: First, one must generate the separate cumulative normals for the states greater and lesser. For each $\mathrm{Co}$, then, the $\operatorname{Pr}$ (uncertain) is given by $1-[\operatorname{Pr}($ greater $)+\operatorname{Pr}($ lesser $)]$. Next, the expected frequency of the three states is obtained for each Co by multiplying these probabilities by the frequency of its occurrence. The sum of these expected frequencies for the states greuter and lesser are then calculated. These sums form the base frequencies of the two responses, dependent only on sensory processes. The total number of responses not accounted for by the sum of these sums is the number of uncertain states entered into. $\mathbf{T}$ is number is then divided hetween two response classes, "Lesser" and "Greater." in a ratio such that the total number of "Lesser" and "Greater" responses will be equalized. For each Co, the expected number of uncertain states is partitioned into the two response classes using the ratio obtained in the previous step. A psychometric function can now be generated. relating the probability of making a "Greater" response to the $\mathrm{Co}$ values. From this psychometric function a PSE can be obtained.

The application of this procedure to the Restle and Levison data first required the estimation of the parameters needed for the gencration of the two cumulative normals. These parameters are $T_{L}, T_{U}$, and the standard deviations of the two functions. Since the two groups in the Restle and Levison experiment both showed a constant error, we first estimated the PSE. This PSE was based on the combined data for the two groups. The empirical proportion of responses obtained by Restle and Levison were averaged from the two groups. The averaged proportions were transformed to $z$ scores, and a straight line was fitted by the method of least squares. The PSE for the combined data was determined in the usual manner by solving the equation of the psychometric function for $z=0$ (sce (ivilford, 1954). The PSE obtained this way was 18.24. The same equation was then solved for $z=.67$ and $z=+.67$ to give us the $T_{L}$ and $T_{U}$. respectively. These are the 25 ill and 75 th percentiles of the psychometric function. respectively, and for the averaged data were 16.27 and 20.21. Tuble I gives the distribution of stimuli for the two groups of the Restle and Levison experiment, the empirical proportion of "Greater" responses for each group as well as the average proportion. ${ }^{3}$

The procedure for getting the standard deviations for the two cumulative normals of the model was more involved, since these distributions must have a standard deviation different from (and smaller than) that of the empirical psychometric function. We have assumed that when the empirical probability for making a "Greater" response to some Co is very high $(\mathrm{Pr}=0.96$ or higher), the probability of a sensory state greater for that Co is very nearly the same. This assumption depends upon the fact that for such a stimulus the state uncertain has a very low probability. Likewise. we assume that if the empirical probability of making a "Lesser" response to some ( $(0)$ is very high, that probability is itself a good estimator of the probability of a sensory state lesser. The method of least squares was used on the upper three points of the combined psychometric functions after proportions lad been transformed to 1. values. This calculation showed that the stimulus value 26.03 had an associated $\operatorname{Pr}($ greater $)=.091$. Taking $\mathrm{T}_{\mathrm{U}}=20.21$ as the stimulus for which $\operatorname{Pr}($ greater $)=0.5$, it was determined that $\mathrm{SD}=2.46$. Similarly, using the lower three points of thr empirical psychometric functions, found $\mathrm{SD}=1.96$. Assuming equal SDs, the arithmetic mean of these two gave us an estimate of 2.21 for the SD of each cumulative normal, Having estinated the 


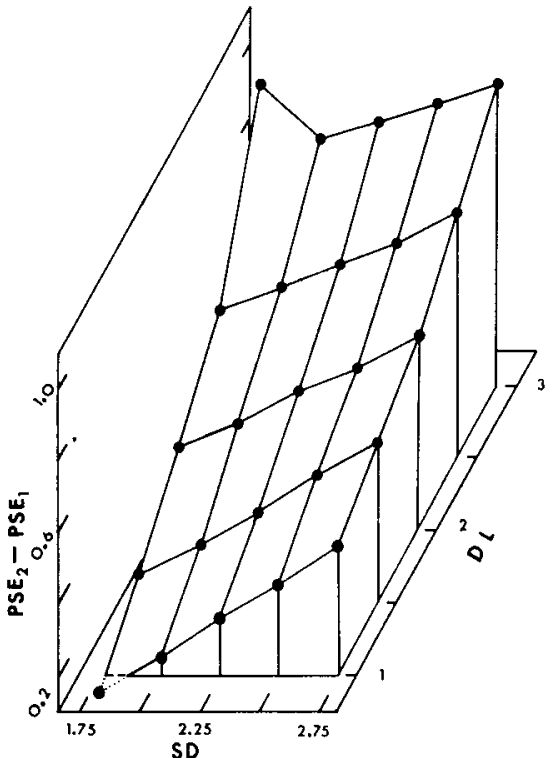

Fig. 5. Difference between the predicted PSEs of Groups 2 and 1 as a function of the standard deviation and the difference threshold.

of these computer runs is that in 24 out of the 25 cases, our model produced a difference between the groups of the Restle and Levison experiment in the same direction as the empirical finding. This makes us confident that the model's response to our original, empirically determined parameter set is not idiosyncratic but, rather, that it is the general case. The differences between the PSEs for the two groups as given by the model for all 25 parameter sets are shown in Fig. 5. The only point that is even slightly in the "wrong" direction is that produced by the combination of the smallest DL and the smallest SD (shown at the front left in the figure). That single aberrant value is instructive in helping us to understand why Restle and Levison erroneously concluded that our RFE model was incompatible with their data. That data point, the only one for which the PSE for Group 2 would be less than that for Group 1 (a difference of 0.04), derives from a conıuter run with parameters of $\mathrm{SD}=1.75$ and $\mathrm{DL}=1.0$ (i.e., $\mathrm{T}_{U}=19.25$ and $\mathrm{T}_{\mathrm{L}}=17.25$ ). When an $\mathrm{SD}=1.75$ is input to the model it yields cumulative normal distributions with steeper slopes than those associated with larger SDs. Moreover, the small difference between $T_{U}$ and $T_{L}$ used in that paremter set yields the least horizontal separation between the cumulative normals. This combination of steepest slope and smallest horizontal separation means that the relatively fow uncertain states will be restricted to those Cos near Std. This singular condition most closely resembles the way in which Restle and Levison applied our model. They make the unlikely assumption that only a single $\mathrm{Co}$, that equal to Std, will yield any uncertain states. Figure 5 shows if uncertain states come to any great extent from other, neighboring stimuli (either by increasing the SD or by increasing the DL, or both) then the model gives more typical results, similar to the findings of Restle and Levison. Indeed, the contours of Fig. 5 make it seem likely that virtually any realistic set of parameters will allow our model to do what Restle and Levison claimed it could not do: predict a higher PSE for their Group 2 than for Group 1.

\section{REMARKS}

As the final argument adduced against RFE theory, Restle and Levison write, "As for the theory that Ss tend to use available responses with equal frequency, we do not find this hypothesis plausible in the face of the cooperativeness of our Ss and the fact that our instructions tell them to make correct comparative judgments [Restle \& Levison, 1971]." This argument is incredible on several counts. The behavior of Ss, including the most cooperative ones is often not consistent with the instructions they receive or even with the way in which they themselves would describe their own performance. In psychophysics, in particular, there are better ways to measure what $S$ does in an experiment than to speculate about his "cooperativeness." Such better ways include the examination of what the $S$ does, i.e., the responses he makes. The fact that our RFE model does a fine job of predicting the data of the Restle and Levison experiment suggests that among the things that their cooperative Ss may do is to tend to make available responses with equal frequency.

We are puzzled by another aspect of the argument that Restle and Levison make against RFE, i.e., that their cooperative Ss were instructed to. make correct comparative judgments on each trial. If Restle and Levison examine their flow chart of their own model (which we assume to be a correct representation of their position), they would realize that their model actually assumes that Ss disobcy the instructions given then! In fact, if one could demonstrate, as Restle and Levison claim in their argument against RFE, that $S$ actually compares the Co and Std presented on each trial, the CAL model would be immediately falsified. It should be recalled that the CAL model asserts that $\mathrm{Ss}$ do not make a judgment of $\mathrm{Co}$ and $\mathrm{Std}$ on each trial, but rather that the $\mathrm{Co}_{0}$ is compared to a weak and very indirect representation of the $\mathrm{Std}$ as it influences the CAL. Thus, if Ss cooperate by actually obeying the Restle and Levison instructions, they would be forced to violate the principles of the Restle and Levison theory.

\section{REFERENCES}

BELL, R. A., \& BEVAN, W. Influence of anchors upon the operation of certain Gestalt organizing principles. Journal of Experimental Psychology, 1968, 78, 670-678.

GUILFORD, J. P. Psychometric methods. New York: McGraw-Hill, 1954.

HELSON, H, Adaptation level theory. New York: Harper \& Row, 1964.

LEVISON, M., \& RESTLE, F. Invalid results from the method of constant stimuli. Perception \& Psychophysics, 1968, 4, 121-122.

RESTLE, F., \& LEVISON, M. Method of constant stimuli: Invalidity to the third power. Perception \& Psychophysics, 1971, 9, 312-314.

SEKULER, R., \& ERLEBACHER, A. The invalidity of "Invalid results from the method of constant stimuli": A common artifact in the methods of psychophysics. Perception \& Psychophysics, 1971, 9, 309-311.

STEVENS, S. S. Problems and methods of psychophysics, Psychological Bulletin, 1958 $55,177 \cdot 196$.

\section{NOTES}

1. We have adopted the convention of beginning the names of sensory states with small letters and placing them in italics. The names of responses, on the other hand, begin with a capital letter and are enclosed in quotation marks.

2. We thank Frank Restle and Moshe Levison for so graciously supplying us with the data of their experiment. We are also grateful to them for sending us a prepublication copy of their paper, which made our response possible.

3. To facilitate future tests of our model, as well as to makc possible idle playing with it, we offer a documented Fortran version of the computer embodiment of RFE. A write-up and program listing may be requested from the second author.

4. Since, in applying the model, the predicted probabilities for the end stimuli are quite extrene, the $\mathrm{z}$ transforms are not very accurate. Because they would be disproportionately weighted in the least-squares fit, we have left out the extreme stimulus at each end in our calculations of the predicted PSEs. We have done this whenever we have calculated predicted PSEs from the model.

5. We have had difficulty determining how Restle and Levison arrived at the PSEs described as "estimates" in their paper. None of the several least-squares fits that we tried gave the values reported by Restle and Levison. Our attempted tits excluded different numbers of data points according to the traditional rules of dropping probabilities beyond various cutoffs. We conclude that the values reported by Restle and Levison were simply read off their graphs, a time-honored, though somewhat dubious, practice (as Note 6 indicates).

6. Restle and Levison use the ratio between their "estimated" PSEs (18.2 and 18.7) to calculate the weight which their model assigns the mean of past Co stimuli. Though on the basis of their earlier experiment they had expected a weight of 0.200 , they report that their data require a weight of 0.289 . If one recalculates that weight by using the PSEs obtained by least squares from their data, the picture changes 


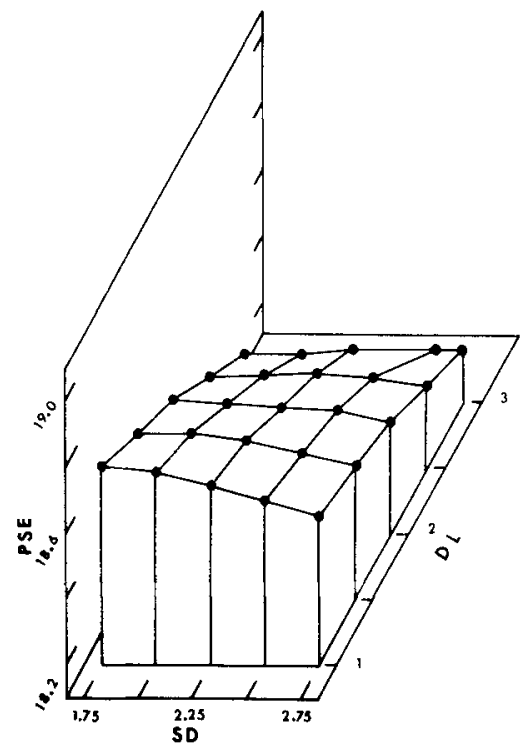

Fig. 3. Predicted PSEs for Group 1 as a function of the standard deviation and the difference threshold.

required three parameters, the application of the model is now straightforward. The values generated by the application of the model are given in Table 2 . The 2 nd and 3rd columns give the values computed from the cumulated normal distributions, given the estimated parameters for $T_{U}, T_{L}$. and $\mathrm{SD}$ derived above. The next column gives the $\operatorname{Pr}$ (uncertain) calculated from $\operatorname{Pr}($ uncertain $)=1-[\operatorname{Pr}($ greater $)+$ $\operatorname{Pr}($ lesser $)]$. The 5 th column gives the number of times each stimulus was presented. When the entry in this column is multiplied by the values in the threc preceding columns, the resulting products are given in the next three columns. These are the expected number of trials on which each of the three sensory states are entered into. The 9 th column gives the expected number of uncertain trials which will be transformed to a response "Greater." These entries are obtained by multiplying the entries in the 8 th column by that ratio which will make the sum of the entrics in the 9 th column plus the sum of the 6th column total to 20 (i.e., half the total number of responses). This last operation represents the application of the RFE principle. The 10th column gives the sums of the entries in the 6 th and 9 th columns. These sums are the expected number of "Greater" responses after the application of the RFE principle. They are converted to probabilities in the 11 th column, giving the predicted psychometric function. The method of least squares was applied to these psychometric functions for the two groups, resulting in a PSE $=18.39$ for Group 1 and a PSE $=18.74$ for Group 2.4 The corresponding values reported by
Restle and Levison are "approximately 18.2 and 18.7." 5 Our own calculations from the Restle and Levison data, using the method of least squares, yields the values of 18.06 and 18.78 .

Restle and Levison contended (1971) that our model would not predict the directional difference between their two groups. The fact remains, however, that our model not only predicts the directional differences of their groups but also comes close to precise prediction of their absolute sizes. Thus our model is not only "logically possible," as previously agreed to by Restle and Levison, but is now shown, by our data, to be empirically true.

To help the reader assess the accuracy of our model in predicting the data of the Restle and Levison experiment, consider how well the CAL theory of Levison and Restle (1968) does the same job. If we are to treat the CAL theory as anything more than a curve-fitting procedure with five weighting parameters free to change at the whim or need of the curve fitter, we should ask how well the equation developed by Levison and Restle (1968) for their original data now fits the data reported by Restle and Levison (1971). When applying their CAL theory to their original set of data, Levison and Restle found that a weight of 0.200 was most appropriate as a description of the contribution which their theory says the mean of all preceding Cos makes to CAL. If the same parameter value were to hold for their more recent experiment (Restle \& Levison. 1971), the PSEs of the two groups should stand in a ratio of 1.019 . Restle and Levison point out themselves that their new data require a change in that weighting parameter in order to provide a better fit to CAL. ${ }^{6}$ It is an interesting anomaly that the PSEs of the two groups in our application of RFE yield precisely that ratio, 1.019 . In other words, we are in the strange position of having a model that produces data slightly more consistent with the predictions of Levison and Restle (1968) than were the actual data of Restle and Levison (1971). Restle and Levison (1971) conclude that "the resuits [of their experiment] agree quite closely with the detailed predictions of the CAL theory." If that is the case, one must surely say that the data of Restle and Levison agree just as well with the detailed predictions of our RFE model.

We have also applied the RFE nodel with only two parameters. If no constant error is assumed, $T_{U}$ and $T_{L}$ would be equidistant from Std and only the $\mathrm{DL}$ would have to be estimated. The SD of the cumulative normals would be the other parameter. Taking $\mathrm{DL}=1.97$, half the distance between $T_{U}$ and $T_{L}$ obtained above and $\mathrm{SD}=2.21$ as before, the

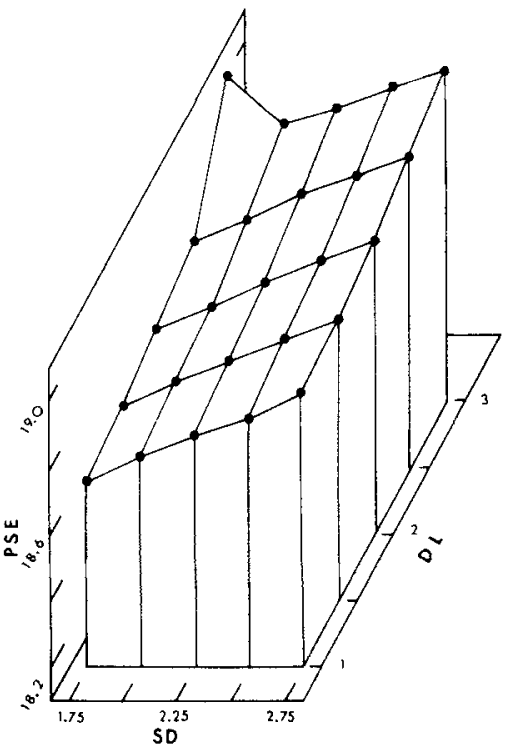

Fig. 4. Predicted PSEs for Group 2 as a function of the standard deviation and the difference threshold.

predicted PSEs become 18.50 and 18.85 for Groups 1 and 2, respectively. Though these values are considerably larger than those obtained earlier and more distant from the empirically obtained PSEs, they are in the right direction. Even with two parameters the model is still adequate.

\section{PARAMETRIC VARIATION OF THE RFE MODEL}

Because of the excellent approxination our model provided to both the direction and magnitude of the Restle and Levison group differences, we were concerned that this outcome was a fortuitous one, peculiar to the particular parameters we had empirically determined for input to the model. To ensure that the values we determined for $T_{U}$, and $T_{L}$, and $S D$ were not unique in their effects upon the model, we embodied the model in a computer program, ${ }^{7}$ which we then ran many times, examining a wide variation of the parameters. We chose 25 different sets of parameters, varying in equal steps around the parameter values we had previously determined as correct, for which we have already given the results. We ran the model with all the factorial combinations of five different SDs $(1.75,2.00,2.25,2.50$, and 2.75) and five different sets of $T_{L}$ and $T_{U}$ $(17.25$ and $19.25 ; 16.75$ and $19.75 ; 16.25$ and $20.25 ; 15.75$ and 20.75: 15.25 and 21.25). The PSEs, calculated as usual by least squares performed on z-transformed proportions, are shown for Group 1 in Fig. 3 and for Group 2 in Fig. 4. In both figures the SDs are arrayed along thc $x$-axis, the DLs along the $y$-axis, and the PSEs on the $z$-axis. The important finding 
drastically, The PSEs taken from our least-squares analysis of the Restle and Levison data are 18.06 and 18.78 for Groups 1 and 2, respectively. The ratio between these two PSEs requires that the weight estimated by Restle and
Levison be increased to 0.415 . This is more than a $100 \%$ change in the value assigned to that weight from one experiment to the next. We cannot agree with the claim of Restle and Levison that their "results agree quite closely with the detailed predictions from CAL theory."

7. Computer time made available from NIH Grant EY-00321.

(Accepted for publication November 16, 1970.) 